\title{
Femtosecond spectroscopic studies of photoinduced electron transfer in MDMO-PPV:ZnO hybrid bulk heterojunctions
}

cursor approach as the MDMO-PPV:TiO ${ }_{2} \mathrm{BHJ}$ cells that were mentioned above. The aim is to investigate the influence of the ratio of $\mathrm{ZnO}$ relative to MDMO-PPV on the polaron generation, transport and recombination processes in the blend.

\section{Experimental}

The preparation of MDMO-PPV:ZnO blend films via the precursor route is described in detail in Ref. [3]. The femtosecond transient absorption setup covers the spectral range from 0.9 up to $3.2 \mathrm{eV}$. Briefly, an amplified Ti:sapphire laser system equipped with two optical parametric amplifiers (OPA) was used to produce a $\sim 100 \mathrm{fs}$ laser pulse train at a repetition rate of $1 \mathrm{KHz}$. The probe beam was provided by white light generated by focusing a small amount of the fundamental beam on a sapphire plate $(0.9-2.7 \mathrm{eV})$ or on a $\mathrm{H}_{2} \mathrm{O}$ cell $(1.1-3.2 \mathrm{eV})$. Measurements were performed such that the pump beam irradiated a larger area of the sample $\left(\sim 0.25 \mathrm{~mm}^{2}\right)$ compared to the probe beam $\left(\sim 0.1 \mathrm{~mm}^{2}\right)$ in order to guarantee a homogeneous optical density throughout the probing area. The instrument response of the setup is $\sim 200 \mathrm{fs}$. During the 


\section{ARTICLE IN PRESS}

1 experiments the samples were kept at $80 \mathrm{~K}$ in a cryostat flowed with nitrogen and under atmospheric pressure. 3

\section{3. Results and discussion}

7 The excitation energy in the femtosecond transient absorption experiments was kept at $2.2 \mathrm{eV}$ to minimize

9 the influence of photo-degradation effects on the measurements and excitons are created. Probing at $2.1 \mathrm{eV}$ reveals

11 the changes in absorption due to either stimulated emission (SE) of the exciton and/or exciton-exciton annihilation

13 (E-EA). In the pristine conjugated polymer a negative signal is observed (wt ratio 1:0, see Fig. 1a) and the

15 transient comprises an ultrafast decay $\sim 500$ fs and a long component with several hundred picoseconds under

17 relatively low excitation intensity $(\sim 0.8 \mu \mathrm{J} /$ pulse $)$. The negative signal at $2.06 \mathrm{eV},(600 \mathrm{~nm})$ was argued to be

19 dominated by SE of the exciton. The ultrafast component of the SE is characteristic of E-EA and the long

21 component is typical of the exciton lifetime. Increasing the excitation intensity $(\sim 2.5 \mu \mathrm{J} /$ pulse $)$ results in the

23 shortening of the ultrafast component to $\sim 280 \mathrm{fs}$. This is in line with the E-EA assignment.

25 Fig. 1b illustrates transients at $2.1 \mathrm{eV}$ for MDMO-PPV:ZnO (wt ratio 1:1). At the low excitation

27 intensity the transient behaves similar to that of pristine MDMO-PPV polymer film as shown in Fig. 1a, except that

29 the relative amplitude of the long component of SE is smaller and the ultrafast component is even faster to

$31 \sim 100$ fs. The weakening of the long component of SE can be explained by the fact that the blend is in fact a mixture of two phases: one is the pristine MDMO-PPV, which is dominant in the areas without $\mathrm{ZnO}$ and the other is MDMO-PPV:ZnO blend phase being dominant in the areas where $\mathrm{ZnO}$ is in the vicinity of MDMO-PPV conjugated polymer chains. In the blend phase electron transfer occurs at the interfaces between MDMO-PPV conjugated polymer chains and $\mathrm{ZnO}$ nanoparticles. The excitons in the blend phase also have contributions to the transient at $2.1 \mathrm{eV}$ and their lifetime will be limited by the electron transfer process from MDMO-PPV to $\mathrm{ZnO}$, which is known to be less than $100 \mathrm{fs}$ when the electron acceptor is PCBM [5]. We assume that the electron transfer time will be similar when the electron acceptor is $\mathrm{ZnO}$. The relative amplitude of the long SE component is therefore smaller compared to pristine MDMO-PPV films (Fig. 1a). Also the time constant of the ultrafast SE component will be shorter than that in the pristine film due to the existence of the excitons of ultra-short lifetime in the blend phase.

Increasing the excitation intensity results in a change in the decay kinetics. As can be seen in Fig. 1b, the signal changes from negative to positive. Apart from SE and E-EA, also polaron absorption is present at the probe energy. This polaron absorption is responsible for the extra component with time constant of $\sim 2$ ps (Fig. 1b). According to the discussion above the signal at $2.1 \mathrm{eV}$ is a superposition of exciton SE and polaron absorption in the blend phase and exciton SE in pristine phase. In the pristine phase the transient at $2.1 \mathrm{eV}$ should reflect merely the feature of pristine polymer similar to Fig. 1a, while in

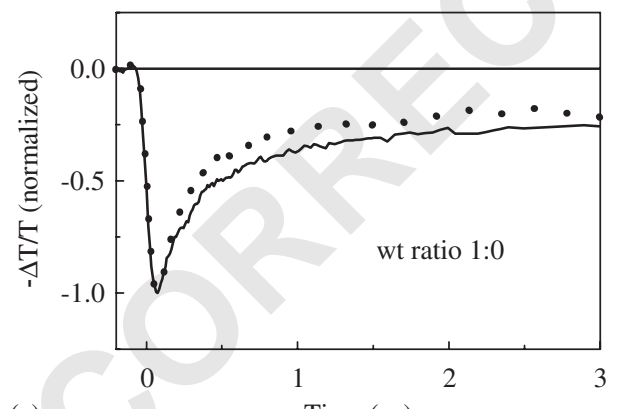

(a)

Time (ps)

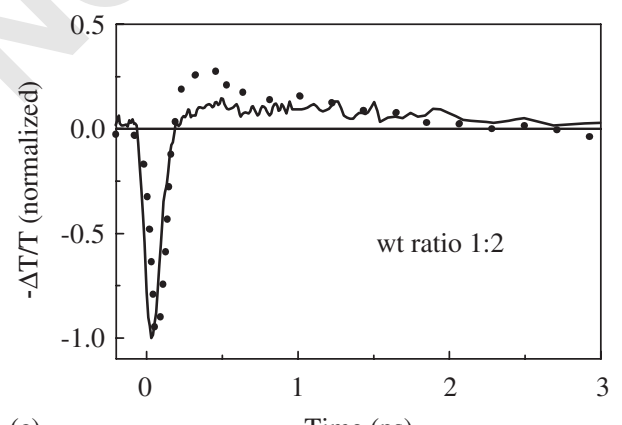

(c)

Time (ps)

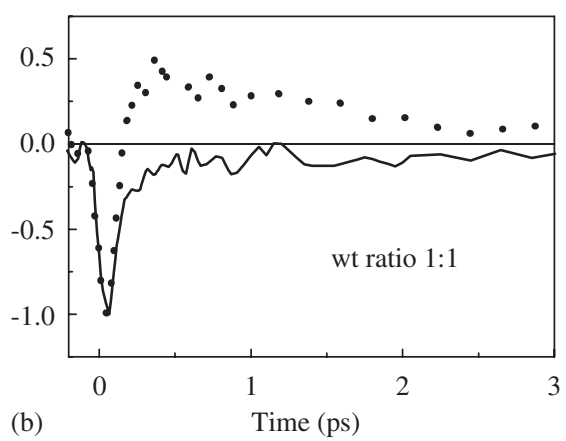

91

93

101

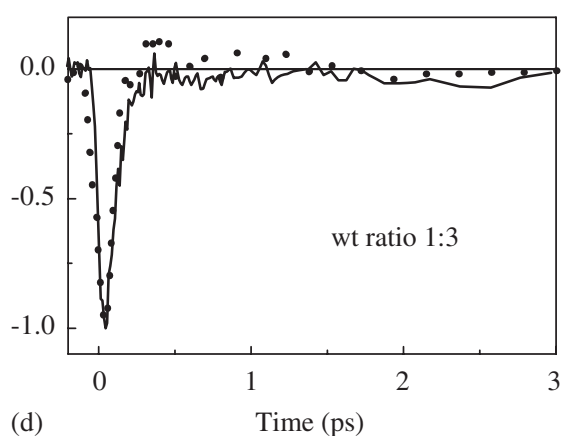

103

105

107

Fig. 1. Kinetics of transient absorption at $2.1 \mathrm{eV}$ of MDMO-PPV:ZnO blend films with excitation at $2.2 \mathrm{eV} . T=80 \mathrm{~K}$. Excitation intensities are $0.8 \mu \mathrm{J}$ 57 pulse (solid curves) and $2.5 \mu \mathrm{J} /$ pulse (dotted curves), respectively. 
1 the blend phase electron transfer occurs and in consequence polarons are generated. The fact that a positive component appears at high excitation intensity reveals the excitation intensity dependence of the polaron generation in the blend phase. This observation suggests that besides the exciton decomposition, E-EA is another channel to 7 generate polaron. Therefore, the polaron number can be regarded as being proportional to the number of excitons,

9 and thus has a linear function of the excitation intensity, while saturation effect manifests itself in an underlinear

11 function of the excitation intensity in the exciton absorption. The short lifetime of the polaron $(\sim 2 \mathrm{ps})$ implies that

13 in the blend phase the polaron cannot diffuse or transport long before it recombines, e.g. with negatively charged $\mathrm{ZnO}$. More $\mathrm{ZnO}$ in the MDMO-PPV:ZnO blend increases the area of blend phase relative to the pristine polymer

17 phase as is reflected in the transients of blend 1:2 shown in Fig. 1c. At relatively low excitation intensity a positive signal already appears with a time constant $\sim 2 \mathrm{ps,} \mathrm{in}$ contrast to the case in blend 1:1 (Fig. 1b).

This might generate the expectation that stronger absorption of the polaron would show up at low excitation intensity if more $\mathrm{ZnO}$ were in the blend. This, however, turns out to be not the case. Higher $\mathrm{ZnO}$ concentration quenches the spectroscopic signature of the polaron. Fig. 1d shows the transient at $2.1 \mathrm{eV}$ of a MDMO-PPV:ZnO blend with weight ratio $1: 3$. It can be seen that only the ultrafast SE is apparent. Neither the long SE component nor the polaron absorption is significant. Disappearance of the polaron absorption may be due to several reasons, e.g. (1) lack of the blend phase or (2) ultrafast back electron transfer. Indeed, the scanning electron microscopic picture of the blend 1:3 shows holes in $\mathrm{ZnO}$ area, while relatively homogeneous structure is shown in the SEM pictures of the blend films 1:1 and 1:2. This would imply that in blend film $1: 3$ the blend phase area has been reduced compared to the blend films 1:1 and 1:2. On the other hand the ultrafast SE $\sim 100 \mathrm{fs}$, differing from the pristine MDMO film, indicates that polarons are generated in the blend film 1:3. Thus absence of the polaron absorption could also be argued to come from the speed up of the back electron transfer, which is consistent with the observation that the excitons in the blend phase live ultrashortly $\sim 100 \mathrm{fs}$. We infer that both factors exist in blend film 1:3.

\section{Conclusion}

In summary, the photogeneration of polarons resulting from electron transfer from MDMP-PPV conjugated polymer chains to $\mathrm{ZnO}$ at $80 \mathrm{~K}$, strongly depends on the weight ratio between MDMP-PPV and $\mathrm{ZnO}$ and on the excitation intensity. It is confirmed from the femtosecond transient absorption kinetics at $2.1 \mathrm{eV}$ that the polarons are generated within the excitation laser pulse $(\sim 100 \mathrm{fs})$ and have a lifetime of $\sim 2 \mathrm{ps}$ in MDMO-PPV:ZnO blends. Both pristine polymer and blend phase coexist in the blend films $1: 1$ and 1:2. Increasing the excitation intensity favors the generation of the polaron. Quenching of the absorption of the polaron in high $\mathrm{ZnO}$ concentration is ascribed to the lack of the blend area and the speed up of back electron transfer.

\section{Acknowledgments}

This work is partly supported by the Dutch Polymer Institute project "Nanocomposite hybrid photovoltaic cells".

\section{References}

[1] H. Spanggaard, F.C. Krebs, Solar Energy Materials \& Solar Cells 83 (2004) 125

[2] C.J. Brabec, N.S. Sariciftci, J.C. Hummelen, Adv. Funct. Materials. 11 (2001) 15.

[3] W. J. E. Beek, L. H. Slooff, Martijn M. Wienk, Jan M. Kroon and René A. J. Janssen, Adv. Funct. Mater, accepted.

[4] N.S. Sariciftci, L. Smilowitz, A.J. Heeger, F. Wudl, Science 258 (1992) 1474.

[5] C.J. Brabec, G. Zerza, G. Cerullo, S. De Silvestri, S. Luzzati, J.C. Hummelen, S. Sariciftci, Chem. Phys. Letters 340 (2001) 232. 